\title{
Percepción de la salud por adultos mayores que realizan actividad física en un parque público de Cali, Colombia
}

\author{
Perception of health by older adults who perform physical activity in a public park \\ in Cali, Colombia
}

\author{
Percepção da saúde pelos idosos que realizam atividade física num parque \\ público em Cali, Colômbia
}

\author{
Lizardo Saavedra Conde', Cecilia Andrea Ordóñez-Hernández², Luis Carlos Granja Escobar , Jessica Castro Hernández \\ 1 Magíster en Salud Pública. Universidad del Valle. Cali. Colombia. lizardo.saavedra@correounivalle.edu.co. oRCID: https://orcid.org/0000- \\ 0002-3383-1965 \\ 2 Doctora en Ciencias de la Salud en el Trabajo. Universidad del Valle. Cali. Colombia. cecilia.ordonez@correounivalle.edu.co. Orcid: \\ https://orcid.org/0000-0001-9296-3768 \\ 3 Doctor en Educación. Universidad Santiago de Cali. Cali. Colombia. luiscarlose@gmail.com. oRciD: https://orcid.org/0000-0002-1546-1542 \\ $4 \quad$ Fisioterapeuta. Universidad del Valle. Cali. Colombia. Jessica.castro@correounivalle.edu.co. oRCID: https://orcid.org/0000-0002-0404-038X
}

Recibido: 28/04/2020. Aprobado: 15/06/2021. Publicado: 14/07/2021

Saavedra-Conde L, Ordóñez-Hernández C, Granja-Escobar LC, Castro-Hernández J. Percepción de la salud por adultos mayores que realizan actividad física en un parque público de Cali, Colombia. Rev. Fac. Nac. Salud Pública. 2021;39(3):e341345. DoI: https://doi.org/10.17533/udea.rfnsp.e341345

\section{Resumen}

Objetivo: Comprender la percepción que sobre la salud tienen los adultos mayores que realizan actividad física en un parque público de Cali, Colombia. Metodología: Se llevó a cabo un estudio de caso cualitativo, con enfoque fenomenológico. El grupo de estudio estuvo conformado por siete adultos que realizan actividad física en el parque, seleccionados por muestreo propositivo por conveniencia. La técnica utilizada fue la entrevista en profundidad. Resultados: Se encontró que la percepción que sobre la salud tienen los adultos mayores es positiva y está relacionada con la independencia, la autonomía, el poder valerse por sí mismos, sentirse útiles y relacionarse con otras personas. Conclusión: La actividad física es utilizada por el adulto mayor para conservar la salud y como un pretexto para interactuar con sus pares, utilizar el tiempo libre y desarrollar tareas en pro de su comunidad

---------Palabras clave: actividad física, adulto mayor, promoción de la salud, Colombia, salud de grupos específicos.

\section{Abstract}

Objective: To understand the perception of health by older adults who perform physical activity in a public park in Cali, Colombia. Methodology: A qualitative case study was carried out with a phenomenological approach. The study group was made up of seven adults who perform physical activity in the park, selected by convenience and purposeful sampling. The technique used was the in-depth interview. Results: It was found that the perception that older adults have about health 
is positive and is related to independence, autonomy, being able to fend for themselves, feeling useful, and interacting with other people. Conclusion: Physical activity is used by the elderly to maintain health and as a pretext to interact with their peers, use their free time, and undertake tasks in favor of their community.

-Keywords: physical activity, elderly, health promotion, Colombia, health of specific groups

\section{Resumo}

Objetivo: Compreender a percepção que os idosos que realizam atividade física num parque público em Cali, Colômbia, têm sobre a saúde. Metodologia: Foi realizada uma pesquisa qualitativa com análise fenomenológica. O grupo de estudo foi formado por sete adultos que realizam atividade física no parque, selecionados por amostragem propositiva por conveniência. A técnica utilizada foi entrevista em profundidade. Resultados: Encontrou-se que a percepção que os idosos têm sobre a saúde é positiva e está relacionada com a independência, a autonomia, o poder de se defenderem por si mesmos, sentirem-se úteis e relacionar-se com outras pessoas. Conclusão: A atividade física é usada pelo idoso para conservar a saúde e como um pretexto para interagir com seus pares, utilizar o tempo livre e desenvolver tarefas em prol da sua comunidade.

---------Palavras-chave: Atividade física, Idosos, Promoção da saúde, Colômbia, Saúde de grupos específicos.

\section{Introducción}

La percepción de la salud de la persona adulta mayor surge de su experiencia y se expresa en sus propios términos y significados. Esta percepción es diversa y susceptible de cambios, en función de elementos que no necesariamente están en relación con el estado físico y funcional [1], sino que dependen de circunstancias personales y contextuales. El juicio que cada adulto mayor posee de su salud, junto con el nivel de la importancia o valoración que le concede, tiene gran influencia en su bienestar, de tal forma que a medida que la persona se considere sana, será capaz de realizar actividades satisfactorias y que influyan en una valoración positiva de la vida [2].

El aumento de la esperanza de vida ha sido un gran avance en la salud pública; no obstante, los individuos están viviendo condiciones que no son las más adecuadas: pobreza, desigualdad, discriminación, abandono y problemas que cada vez enfrentan las personas adultas mayores y comprometen su percepción de la salud, y son más susceptibles a enfermar y, por tanto, a aumentar su demanda de servicios de salud [1]. Se ha identificado que la percepción negativa de la salud se encuentra relacionada con la enfermedad [1,3]; el deterioro de la condición física [4], psicológica [5] o social [6], y que afecta el bienestar subjetivo y la calidad de vida en el adulto mayor [2].

La actividad física genera una percepción positiva de la salud en el adulto mayor, mejorando sus condiciones físicas, psicológicas y sociales [7-10] y a pesar de que sus múltiples beneficios son conocidos, se ha reportado significativamente que a medida que las personas envejecen, realizan menos actividad física [11].

Principalmente, las investigaciones han estudiado la percepción de la salud como un indicador subjetivo del estado de salud $[4,12,13]$, lo que las ubica en una mirada biopsicosocial basada en una perspectiva de funcionamiento humano, independencia y autonomía, que dimensiona la interacción entre la persona y los factores contextuales físicos y socioculturales en los que se desarrolla la cotidianidad del adulto mayor, sin considerar como una particularidad la práctica de la actividad física [7]. Sin embargo, el envejecimiento no necesariamente significa una disminución de las funciones físicas, cognitivas y sociales, ya que este no se produce de manera homogénea, sino de modo particular desde su historia de vida, lo que incide en su percepción de la salud. Todo ello puede aportar a la comprensión de su comportamiento en salud y generar estrategias efectivas que contribuyan a trascender la visión del envejecimiento como problema, y a la mirada del envejecimiento activo como estrategia generadora de bienestar y salud, así como al aumento de la participación de estas personas en la práctica de la actividad física.

Es relevante abordar la percepción de la salud en el adulto mayor que practica actividad física desde una perspectiva fenomenológica, es decir, a partir del conocimiento que genera su propia experiencia, en tanto permite profundizar sobre lo que piensa, siente y da sentido a su vida cotidiana. Además, el enfoque fenomenológico aporta el punto de vista de propio sujeto y permite la oportunidad de diversificar los conocimientos que sobre el tema se han descrito ampliamente desde la perspectiva biomédica (la que, por el contrario, privilegia la postura del investigador), visibilizando las subjetividades en los adultos mayores que interactúan con el entorno del parque público y han desarrollado una particular visión sobre su propia salud [14-15].

Para Merleu Ponty, la comprensión del mundo y de cualquier fenómeno humano ocurre en la intersección 
entre las propias experiencias con las de los semejantes en "variados horizontes vivenciales, como el espacio, el tiempo, el cuerpo y la propia inter-subjetividad" [14, p. 248]; de esta manera, el mundo la realidad se constituye en la relación mutua de sujeto y objeto .

La fenomenología describe las vivencias humanas, a través de la percepción como contacto antepredictivo, prerreflexivo con el mundo. Lo "antepredictivo" se refiere a entender el mundo no a través de los conceptos, sino por medio de los significados dados a las experiencias vividas. La percepción es un contacto que nos lleva a un encuentro íntimo con el mundo, por lo que es una experiencia situada, subjetiva y temporal.

Este es un artículo que ha pretendido describir una percepción natural de la salud de los adultos mayores que realizan actividad física en la cotidianidad en un parque barrial desde sus vivencias, sensaciones, sentimientos, opiniones y conocimientos de estas personas. En este sentido, ha buscado dar voz desde su encuentro con la realidad, lo que ha permitido que emerjan sus particulares miradas sobre la percepción de la salud.

Así, el objetivo de la investigación fue entender la percepción que sobre la salud tienen los adultos mayores que realizan actividad física en el parque La Virgen, del barrio Villas de Guadalupe, de la Comuna 17 de la ciudad de Cali. De este modo, se espera que este estudio aporte a la "Política colombiana de envejecimiento humano y vejez 2015-2024" [16].

\section{Metododología}

El diseño fue cualitativo. El tipo de estudio fue un estudio de caso fenomenológico, el cual explora un fenómeno en su propio escenario [17]. La fenomenología, por su parte, es un enfoque teórico del paradigma interpretativo, que parte del conocimiento de la experiencia subjetiva de los hechos vividos tal como se perciben [18], y puede analizarse a la luz de los cuatro existenciales: el cuerpo vivido, el tiempo vivido, el espacio vivido y las relaciones humanas vividas [14-15]. Según Ponty, la única manera de existir para cualquier mundo corpóreo es a través del tiempo y en el espacio, inmersos en la voluntad humana y en la interacción con los otros, lo que configura nuestra [15].

\section{Pasos para el estudio de caso}

Chetty [17] describe los pasos que deben seguirse para llevar a cabo un estudio de caso:

1. Definición de la unidad de análisis: la unidad de análisis simple corresponde a los adultos mayores que realizan actividad física en un parque público de Cali y quienes participaron en la investigación.

2. Selección de la muestra: el grupo de estudio estuvo conformado por siete adultos mayores, que fueron seleccionados mediante un muestreo por conveniencia. En este tipo de muestreo, los informantes no son seleccionados al azar, sino según la facilidad de acceso. Los criterios de inclusión fueron practicar actividad física en el parque La Virgen, manifestar interés de participar en el estudio, tener facilidad de conversación y la disposición para abordar el tema en cuestión.

3. Recolección de información: como técnica de recolección de información, se hicieron dos entrevistas en profundidad a cada participante, con duración promedio de 1 hora y media cada una. Se explicó el objetivo del estudio, la intencionalidad de la conversación y se solicitó el consentimiento informado por escrito. Las entrevistas se realizaron en el parque o en las casas de los participantes, según su conveniencia.

Como instrumentos de recolección se diseñaron:

a. Protocolo de reclutamiento: consiste en la planeación y la descripción anticipada de los pasos que deben seguirse antes de llevar a cabo las entrevistas, con el fin de estandarizar el proceso. En ese momento, se analizan el número de sujetos que se tiene planeado potencialmente entrevistar; los criterios de selección; la descripción de cómo, cuándo y dónde se llevará a cabo la invitación; el lugar de la reunión; la duración mínima promedio; los momentos de la entrevista: la preentrevista, la entrevista y el cierre. También contempla la presentación del proyecto, la reiteración del consentimiento informado y la formulación de las normas básicas de la entrevista, así como las condiciones de grabación y de registro, y los materiales necesarios.

b. Guía de entrevista: consiste en un cuestionario de preguntas, agrupadas por los ejes temáticos, que permiten encuadrar los temas a tratar. Estas preguntas no se constituyen en un guion y pueden no ser formuladas como están escritas, ni llevan un orden específico. Se usan para recordar al entrevistador los temas de interés, y aquellos que son necesarios abordar durante la entrevista, la cual debe parecerse lo más posible a una conversación natural, en una ambiente de confianza.

4. Análisis de la información: las entrevistas finalizaron cuando el entrevistado consideró que se había agotado todo lo que podía decir sobre el tema. Se efectuaron en total catorce entrevistas, que fueron registradas en una grabadora digital y transcritas en su totalidad como una sola unidad hermenéutica en el programa de análisis cualitativo Atlas Ti 8.0 [19]. Para el análisis de la información, se utilizó el análisis cualitativo propuesto por Taylor [20], el cual se basa en tres momentos: 
a. Descubrimiento: consiste en buscar de modo exhaustivo los temas planteados por los entrevistados.

b. Codificación: consiste en el agrupamiento de los contenidos encontrados en la entrevista, según ideas, conceptos e interpretaciones mencionadas por los entrevistados. En este momento, se hace un análisis más detallado que permite organizar y agrupar tanto categorías como conceptos emergentes de lectura y relectura.

c. Relativización o interpretación de los datos: $\mathrm{Se}$ analizan los datos en relación con el contexto en el que fueron recogidos.

\section{Aspectos éticos}

Las personas que participaron en el estudio dieron su consentimiento de manera escrita y voluntaria para hacer parte de la investigación. A cada persona se le dio la información adecuada y concreta sobre el método del estudio. De la misma manera, quedó claro que la persona podía desistir de participar en el estudio o retirarse en cualquier momento.

Se preservó la confidencialidad de la identidad del informante.
Esta investigación se realizó bajo los principios éticos de la Declaración de Helsinki [21]; se clasificó en riesgo mínimo según la Resolución 8430 de 1993 del Ministerio de Salud en Colombia [22] y se aprobó por el Comité Institucional de Revisión de Ética Humana de la Facultad de Salud de la Universidad del Valle, según el Acta de aprobación 007-016 del 23 de noviembre de 2018.

\section{Resultados}

Los participantes fueron 7 adultos mayores, 4 mujeres y 3 hombres, con edades entre los 62 y los 84 años. Respecto a sus estudios, 2 personas finalizaron su bachillerato y 5 su educación universitaria. Además, 3 son pensionados, 2 amas de casa, uno es un enfermero y el otro un profesor. Ellos han utilizado el parque entre 6 y 20 años.

En la Tabla 1 se muestran los datos sociodemográficos de los adultos mayores participantes en la entrevista en profundidad.

A continuación se describen los resultados en tres fases: descubrimiento, codificación y relativización.

Tabla 1. Datos sociodemográficos de los adultos mayores participantes en la entrevista en profundidad

\begin{tabular}{lcclcc}
\hline Participante* $^{*}$ & Sexo & Edad años & Nivel educativo & \multicolumn{1}{c}{ Ocupación } & Tiempo de uso del parque (años) \\
\hline Samán & Masculino & 62 & Universitario & Enfermero & 26 \\
Gualanday & & 66 & & Profesor colegio & 19 \\
Ciprés & 70 & & Profesor pensionado & 20 \\
Jazmín nocturno & Femenino & 70 & Secundaria & Ama de casa & 10 \\
Hortensia & 72 & Universitario & Enfermera pensionada & 8 & 8 \\
Coral & 72 & & Profesora pensionada & 6 \\
Duranta & 84 & Secundaria & Ama de casa & \\
\hline
\end{tabular}

* Los nombres expuestos corresponden a plantas presentes en los parques de Cali y coinciden solamente con la inicial del nombre verdadero de los participantes.

\section{Primera fase: descubrimiento}

Los adultos relatan una forma de relacionarse con el parque más allá de la realización de ejercicio. El parque favorece la interrelación entre las personas que lo habitan y motiva en ellos objetivos comunes, referentes al cuidado y el mantenimiento del mismo. El cuidado de árboles, las plantas y el embellecimiento en general de todas las zonas, así como la limpieza, la buena presentación del parque y su buen uso forman parte de las actividades cotidianas de algunos entrevistados.

[...] en los momentos libres que no estoy ocupado disfruto mucho de sentarme en el parque, de compartir con los vecinos un café con un pandebono, o una cerveza, o un jugo, o jugar un parqués, [...] podemos compartir, podemos charlar, podemos reírnos, echar cuentos, contar anécdotas entre vecinos [...] (Samán).
[...] en lo que yo puedo colaborar, la siembra del jardín, las matas y mirar que la gente que viene aquí al parque lo use adecuadamente, sobre todo en la cuestión de la basura [...] que el parque se vea bien presentado, bien aseado [...] (Jazmín nocturno).

La caminata individual o grupal es vivida como una experiencia corporal saludable; genera conciencia del propio cuerpo al respirar, al tiempo que se interactúa con la naturaleza. En algunos meses del año, estas actividades son orientadas por fisioterapeutas y en su ausencia surgen líderes que asumen su rol.

[...] el caminar es una forma de verificar que la salud está bien, en el caminar uno percibe su ritmo cardíaco, ehh, percibe su nivel de fatiga, uhmm, su nivel de vitalidad [...] (Gualanday). 
El gusto por caminar gira en torno a la vida social, en algunos casos, desde la juventud, y en otros, motivados por vecinos o amigos que los convocaron a conformar un grupo o simplemente a salir a hacer ejercicio en compañía.

$[\ldots]$ otro beneficio de mi ejercicio es que me relaciono con personas, conozco a otras personas; entonces, me hace más sociable, me relaciono con personas que nunca he conocido y ya entablamos una amistad [...] (Jazmín nocturno).

La práctica de ejercicio está ligada a la preservación del cuerpo y al mantenimiento de la salud como componentes primordiales para mantener la independencia. Reconocen la salud como una condición de existencia; al perderla, se pierde la autonomía.

La salud es reconocida como un conjunto de factores corporales, emocionales y mentales que se influyen de manera constante y bidireccional, e influyen en la construcción de vínculos sociales y afectivos.

[...] pues la salud es el estado en óptimas condiciones físicas y mentalmente de una persona (Samán).

[...] ¡Jumm! Pues, es lo más importante; si uno no tiene salud, ¿pa' qué vida? Yo pienso eso. Le pido a Dios que me dé salud, porque si no, que me lleve; yo no quiero estar enferma, yo no quiero estar en una cama estorbando [...] (Coral).

En algunos casos, los adultos consideran el ejercicio como reemplazo de la terapéutica médica. Para ellos, con la actividad física no hay necesidad de tomar tantos medicamentos y por propia decisión disminuyen la dosis o los suspenden. Asumen su "cuerpo soberano" y ejercen autonomía sobre él y sobre su salud.

[...] no, hermano, es que uno es el real dueño de su vida. Los seres humanos, entre más autónomos seamos, mejor; no podemos entregarle la soberanía de nuestro cuerpo al médico, ¿y qué tal si el médico se equivoca? ¡Quién mejor que uno para interpretar su propio cuerpo! Entonces, por ejemplo, le estoy bajando a la otra droga, y vamos a ver cómo me va con los exámenes, por supuesto [...]. Yo lo que digo es que los seres humanos tenemos que tratar que uno sea el dueño de su vida, de sus emociones, de sus pensamientos, de sus imágenes y, por supuesto, de lo que refleja su cuerpo, porque el cuerpo es un receptor de todo eso; pero uno mismo puede generar sus propios mecanismos de equilibrio y de armonización. El cuerpo es un aparato extraordinario, maravilloso (Gualanday).

Cada adulto mayor, de acuerdo con su percepción de la salud, ha tenido sentimientos de satisfacción, felicidad y agrado. Asimismo, se han sentido saludables, útiles e independientes en sus actividades diarias. Estas emociones y valores han sido tejidos al interactuar con sus pares en las actividades grupales que organizan tanto en beneficio del parque como a nivel social.

[...] yo reconozco que he mejorado mi salud, he superado algunas deficiencias de salud que tenía, por medio del ejercicio, por medio de las relaciones de amistad que he tenido con la gente, por medio del afecto que uno recibe de la gente, por el agradecimiento que le da la gente todos los días. Eso es salud y eso es bienestar [...] (Hortensia).

[...] primero, me siento saludable, porque tengo un nieto que en este momento... él me inspira mucha estabilidad, no económica, sino emocionalmente; luego, vivir al lado del parque, donde respiro aire puro; eso contribuye al bienestar de la salud y estar bien. Tener vecinos, buenos vecinos. Esa comunicación social con las personas, todas las cosas de mi entorno me hacen sentir saludable [...] (Jazmín nocturno).

\section{Segunda fase: codificación}

En esta fase, los fragmentos de las entrevistas fueron agrupados según su correspondencia y similitud. Se creó una lista de códigos con los temas que emergieron de los datos, los cuales fueron definidos y agrupados en categorías, de acuerdo con cada uno de los existenciales básicos (véase Tabla 2). De esta manera, el existencial de las "relaciones humanas vividas" estuvo conformado por las categorías "Vecinos", "Grupo de adulto mayor" y "Personal de la salud", las cuales fueron coincidentes en todos los informantes. El existencial "cuerpo vivido" estuvo conformado por las categorías "Cuerpo saludable", "Cuerpo soberano" y "De la enfermedad a la salud del cuerpo". Por su parte, el existencial "Tiempo vivido" estuvo constituido por las categorías "Sentirse saludable en el tiempo" y "Tiempo libre", y el existencial "espacio vivido", por la categoría "Parque sentido".

Este proceso de codificación se realizó teniendo en cuenta el contexto, ajustando los códigos y las categorías lo mejor posible a la realidad.

\section{Tercera fase: relativización o interpretación}

En esta etapa se interpreta la información, describiendo el contexto en el que fueron obtenidos los datos y se capturan las situaciones que resultaron trascendentales para los informantes a la luz de los cuatro existenciales [14-16].

\section{Relaciones humanas vividas}

El adulto mayor realiza actividades sociales con sus vecinos en el parque, como una manera de compartir, tejer relaciones y generar vivencias comunes en él. Estas actividades le permiten mantenerse vigente y útil en su comunidad, haciendo uso de sus habilidades motoras, comunicativas y sociales. 
Tabla 2. Categorías y códigos por cada uno de los existenciales fenomenológicos básicos

\begin{tabular}{|c|c|c|c|}
\hline Existencial & Categoría & Código & Definición \\
\hline \multirow{7}{*}{$\begin{array}{l}\text { Relaciones } \\
\text { humanas } \\
\text { vividas }\end{array}$} & \multirow[b]{2}{*}{ Vecinos } & Actividades sociales & $\begin{array}{l}\text { Acciones realizadas por el adulto mayor con sus vecinos } \\
\text { del parque }\end{array}$ \\
\hline & & Valores & $\begin{array}{l}\text { Capacidades desarrolladas por el adulto mayor, que } \\
\text { favorecen la convivencia en el vecindario ubicado } \\
\text { alrededor del parque }\end{array}$ \\
\hline & \multirow{4}{*}{$\begin{array}{l}\text { Grupo de adulto } \\
\text { mayor }\end{array}$} & Actividad física y salud & $\begin{array}{l}\text { Todo movimiento utilizado para promover relaciones } \\
\text { interpersonales y es percibido por la persona como } \\
\text { saludable }\end{array}$ \\
\hline & & $\begin{array}{l}\text { Relaciones } \\
\text { interpersonales }\end{array}$ & $\begin{array}{l}\text { Interacción que establece la persona mayor con } \\
\text { los vecinos, amigos y con personas del grupo, } \\
\text { para compartir experiencias, sentimientos saberes, } \\
\text { comportamientos }\end{array}$ \\
\hline & & Valores & $\begin{array}{l}\text { Capacidades en los adultos mayores pertenecientes al } \\
\text { grupo "Nueva Vida", que favorecen la convivencia en el } \\
\text { parque }\end{array}$ \\
\hline & & Liderazgo & Capacidad para dirigir la actividad física \\
\hline & Personal de la salud & $\begin{array}{l}\text { Relación con el } \\
\text { profesional de la salud }\end{array}$ & $\begin{array}{l}\text { Interacción positiva o negativa con el profesional de la } \\
\text { salud }\end{array}$ \\
\hline \multirow{6}{*}{ Cuerpo vivido } & \multirow{2}{*}{ Cuerpo saludable } & Cuerpo en movimiento & $\begin{array}{l}\text { Es todo movimiento corporal realizado por el adulto } \\
\text { mayor en el parque }\end{array}$ \\
\hline & & Cuerpo consciente & $\begin{array}{l}\text { Percepción de control físico y emocional, a través de la } \\
\text { respiración y el caminar }\end{array}$ \\
\hline & \multirow[b]{2}{*}{ Cuerpo soberano } & Interpretación del cuerpo & $\begin{array}{l}\text { Es la comprensión que el adulto mayor hace de las } \\
\text { señales corporales que se dan en su cuerpo }\end{array}$ \\
\hline & & El cuerpo que decide & $\begin{array}{l}\text { Autonomía reclamada por la persona adulta mayor } \\
\text { para decidir sobre su cuerpo y las acciones a seguir } \\
\text { respecto al uso de la actividad física, los alimentos y los } \\
\text { medicamentos }\end{array}$ \\
\hline & \multirow{2}{*}{$\begin{array}{l}\text { De la enfermedad a } \\
\text { la salud del cuerpo }\end{array}$} & $\begin{array}{l}\text { La enfermedad como } \\
\text { camino a la actividad } \\
\text { física }\end{array}$ & $\begin{array}{l}\text { Experiencia corporal que contribuye a la realización de la } \\
\text { actividad física en el parque }\end{array}$ \\
\hline & & Miedo al medicamento & $\begin{array}{l}\text { Sentimiento negativo frente al efecto de los } \\
\text { medicamentos en el cuerpo }\end{array}$ \\
\hline \multirow{3}{*}{ Tiempo vivido } & \multirow{2}{*}{$\begin{array}{l}\text { Sentirse saludable } \\
\text { en el tiempo }\end{array}$} & Pasado & $\begin{array}{l}\text { Sentimiento positivo que tiene el adulto mayor acerca de } \\
\text { su propia salud en el tiempo pasado }\end{array}$ \\
\hline & & Presente & $\begin{array}{l}\text { Sentimiento positivo que tiene el adulto mayor acerca de } \\
\text { su propia salud en el tiempo presente }\end{array}$ \\
\hline & Tiempo libre & Tiempo libre & $\begin{array}{l}\text { Tiempo para estar solo o en compañía en el parque, y } \\
\text { salir de la rutina }\end{array}$ \\
\hline \multirow[t]{2}{*}{ Espacio vivido } & \multirow[t]{2}{*}{ Parque sentido } & El Edén & $\begin{array}{l}\text { Es el espacio de encuentro e interacción de las personas } \\
\text { adultas mayores }\end{array}$ \\
\hline & & Cuidado del parque & Sentimiento y acción de cuidado del parque \\
\hline
\end{tabular}

Para el adulto mayor, las actividades sociales son la posibilidad de encontrarse, dialogar, ser reconocido, salir de su hogar y compartir con otros pares, exponer sus puntos de vista sobre situaciones no solo del parque, sino también de otros contextos de su vida.

El adulto mayor que asiste al parque utiliza la actividad física como medio para promover sus relaciones interpersonales, no solo como beneficio individual, sino además como un pretexto para establecer una conexión social que favorece la percepción sobre su salud y le permite reconocerse como sujeto válido dentro de un espacio social.

La relación con los profesionales de la salud es de confianza; aceptan de buen modo las recomendaciones 
que les hacen cuando estas incluyen indicaciones sobre la actividad física y la alimentación como parte de su estilo de vida, y se oponen cuando las acciones se centran únicamente en el uso de medicamentos y no sienten que están contextualizados con su realidad.

[...] en los momentos libres que no estoy ocupado disfruto mucho de sentarme en el parque, de compartir con los vecinos un café con un pandebono, o una cerveza, o un jugo, o jugar un parqués, [...] podemos compartir, podemos charlar, podemos reírnos, echar cuentos, contar anécdotas entre vecinos [...] (Ciprés).

[...] bueno, en el parque, lo escogimos como un centro de encuentro. Todas las personas que nos encontramos allí o nos reunimos allí, somos personas adultas que de una u otra forma necesitamos ocupar el tiempo libre, necesitamos crear más amigos y necesitamos como cambiar, hablar con la gente y de esa manera, pues, dejar la rutina, y de esa manera mejorar el estrés $[\ldots]$ (Hortensia).

[...] no, hermano, es que uno es el real dueño de su vida. Los seres humanos, entre más autónomos seamos, mejor; no podemos entregarle la soberanía de nuestro cuerpo al médico, ¿y que tal si el médico se equivoca? Quién mejor que uno para interpretar su propio cuerpo $[\ldots]$ (Ciprés).

\section{Cuerpo vivido}

Para el adulto mayor, el cuerpo saludable es la expresión del cuerpo en movimiento, activo y funcional, que realiza actividad física en busca del cuidado de su salud. En este sentido, esta práctica voluntaria se hace de manera individual o grupal, según el interés del participante.

A través del cuerpo en movimiento se establecen vínculos en el parque y se llevan a cabo las actividades de la vida diaria, con independencia.

[...] Pues, actividad física es todo en lo que usted produce movimiento, como el cuerpo, caminar, hacer oficio, salir y entrar todo lo que te mueva, actividad física, ejercicios [...] (Jazmín nocturno).

El realizar ejercicio les permite interpretar las señales corporales y tomar decisiones y acciones en torno a su salud, como la disminución del uso de medicamentos y la regulación de la alimentación, el aumento o la disminución de actividad etc., puesto que la presencia de la enfermedad es una de las principales indicaciones para la práctica de actividad física, ya sea por recomendación médica o por prevención de sus consecuencias.

El cuerpo saludable presupone, en primera instancia, un encuentro autoconsciente de sentimientos, experiencias y comportamientos de cada adulto mayor, para luego exteriorizar un cuerpo en movimiento autocrítico y autorregulador de su salud.
[...] hace como unos 3 años empecé a tener problemas de azúcar alta; entonces, el doctor me dijo que "yo de usted, haría lo que fuera para no empezar a tomar medicamentos”. Yo le dije: “¿Qué es lo más indicado para controlar y bajar este problema?". "Yo le recomiendo la dieta y caminar 1 hora diaria y hacer sus ejercicios normales". Yo empecé a caminar 1 hora diaria y eso hace que el azúcar se controló y no tengo necesidad de tomar nada [...] (Hortensia).

\section{Tiempo vivido}

Los adultos mayores evocan la práctica del ejercicio físico en varias etapas de su ciclo vital. La mayoría de quienes ahora son activos físicamente, lo fueron también en su juventud.

En la actualidad, este grupo de personas dispone de gran cantidad de tiempo libre y una de las maneras de utilizarlo efectivamente es mediante la actividad física en el parque, lo que además les facilita otro tipo de actividades recreativas y comunitarias con sus pares.

[...] pues, gracias a dios cumplí 70 años y he sido una persona saludable $[\ldots]$ yo he sido muy aliviada, muy sana [...] (Jazmín nocturno).

\section{Espacio vivido}

Los adultos mayores consideran el parque como un espacio relevante para sus vecinos y residentes; es un espacio apreciado como de gran valor, "algo sagrado", al que reconocen como saludable, agradable, abierto, bello, que les inspira a respirar y moverse. Está rodeado de naturaleza y árboles, que exigen al mismo tiempo cuidados, atención y mantenimiento, responsabilidades que están dispuestos a asumir desde su trabajo y participación comunitaria, pues lo sienten como propio.

Este espacio tiene alto valor significativo en la vida cotidiana de los adultos mayores; les permite expresarse, realizar acciones saludables y construir relaciones afectivas con sus pares.

[...] el parque es un sitio, es un área muy importante para las personas que están cercanas o las que están a poca distancia. Por ahí se puede uno recrear, ahí se puede hacer cantidad de actividades en pro del bienestar físico y emocional de cada persona, de muchas personas [...] (Samán).

[...] el parque es verde, el parque es un pequeño pulmón. Nosotros no hacemos conciencia de esas cosas, pero eso es supremamente importante. Si quizá alguien se pusiera a medir de qué manera el parque alivia la temperatura, pues posiblemente encuentra una relación, y sí, es algo muy micro, pero las casas que están alrededor del parque con toda seguridad sienten ese aporte que les da el parque. Yo estoy seguro que son las casas más frescas del entorno (Gualanday). 
[...] es algo que tengo para tener mi salud, es como algo sagrado, se le convierte a uno como algo sagrado, es el lugar, es una parte muy especial [...] por lo que allí se formó el grupo, pues a mí siempre me ha interesado es el ejercicio. El grupo es para hacer eso, esa es la idea $[\ldots]$ (Duranta).

La percepción de la salud se conforma a partir del disfrute del parque, no solo como espacio de encuentro, sino también como espacio físico, seguro, tranquilo, en el que hay contacto con la naturaleza, en un microclima diferente que se percibe como saludable y que permite el desplazamiento con facilidad por cualquiera de sus áreas, debido a su topografía y a la ausencia de barreras físicas.

El hábito de la práctica de actividad física ha sido construido a través del tiempo y en el momento actual está ligado a exigencias médicas y a la experiencia de sentirse saludable.

\section{Discusión}

Los adultos mayores que realizan actividad física en el parque tienen una percepción positiva de su salud, asociada a diversas formas de entender su cuerpo en interacción con el entorno físico y social del parque. Algunas de las personas entrevistadas entienden la percepción de su salud desde la funcionalidad biopsicosocial del cuerpo, que les permite realizar actividades en el parque con independencia y autonomía.

En nuestro análisis, la capacidad de moverse y sentirse activo representa la satisfacción de ser útil y estar vigente en el tiempo y el espacio. Esto es consistente con estudios cualitativos que relacionan el significado de la salud con la capacidad de poder hacer todas las actividades de la vida diaria de manera independiente [23].

Así mismo, el compartir, tener amigos y recibir afecto contribuyen al mantenimiento de la salud y permiten constituir redes de apoyo, en las que se promueven valores como la solidaridad y la cooperación. La salud se relaciona con sentirse útil y participar de acciones apreciadas por sí mismo y por las personas de su entorno, como cuidar el parque, efectuar la limpieza, hacer actividades sociales para el recaudo de fondos, participar de las reuniones sociales, apoyar con el cuidado de los nietos y la familia, visitar a los enfermos, etc., Estas actividades les otorgan a los adultos mayores la posibilidad de mantenerse vigentes, desempeñando un rol importante para su familia y comunidad. En concordancia, otros estudios confirman la importancia de socializar la persona adulta mayor con sus vecinos y comunidad local para su buena salud física y mental [23-24].

La percepción de la salud también es vista como una experiencia personal de autorreconocimiento, de conexión consigo mismo, para sentir y conectarse con su cuerpo de manera consciente y autónoma, en actividades como la respiración y el caminar. Es una experiencia que interpreta su cuerpo y toma decisiones sobre su salud. Este es un cuerpo soberano que reconoce el parque como un espacio que le contribuye a la responsabilidad sobre su propia salud. En este sentido, Tuohy menciona la autonomía como derecho a la autodeterminación y al control de la propia vida [25]. Ebrahimi et al. [7] nombra el autorreconocimiento como elemento constitutivo de la salud en el adulto mayor. Seah et al. resaltan el valor intrínseco de la salud y el cuidar de uno mismo como una responsabilidad personal [26].

El parque, como espacio vivido a través de la actividad física, está ligado a experiencias individuales y comunitarias, que relatan sentimientos, emociones y vivencias del sentirse saludable, lo que es congruente con el estudio de Wondolowski y Davis: es un espacio de significado particular, configurado por la historia de la actividad física personal, que contribuye a la percepción positiva de la salud [27]; y en el caso de las experiencias comunitarias, al configurarse en una construcción subjetiva compartida entre las personas que hacen uso de un mismo espacio [28].

Las limitaciones del estudio están relacionadas con el hecho de pretender entender la percepción de la salud de adultos mayores que realizan actividad física en un solo parque público barrial, por lo que los resultados pueden enriquecerse y diversificarse desde el entendimiento de otras experiencias vividas en otros parques.

\section{Conclusión}

La percepción de salud para el adulto mayor está relacionada con la posibilidad de mantener la independencia y la autonomía en el desarrollo de las actividades de la vida diaria, y la actividad física como un pretexto para interactuar con sus pares, utilizar el tiempo libre y desarrollar tareas en pro de su comunidad. El parque es reconocido como un territorio de gran valor simbólico, que invita a la práctica de actividad física cotidiana y al establecimiento de vínculos entre sus visitantes, aspectos que deben ser reconocidos y valorados desde la salud pública como elementos claves de la promoción de la salud.

\section{Agradecimientos}

A la Universidad del Valle, Facultad de Salud, Escuela de Salud Pública, Programa de Maestría en Salud Púbica; y en particular a las personas adultas mayores participantes del estudio quienes compartieron su experiencia vivida. 


\section{Conflicto de interés}

Los autores no tienen ningún conflicto de interés

\section{Fuente de financiación}

Este trabajo no recibió financiación de entidades públicas, privadas o sin ánimo de lucro

\section{Declaración de responsabilidad}

Declaración de responsabilidad: Los autores somos responsables del contenido, validez y veracidad de la información escrita en este articulo.

\section{Declaración de autoría}

Declaración de autoría: Todos los autores cumplen los siguientes criterios:

“a).- Contribuir de manera sustancial a la concepción o diseño del artículo o a la adquisición, análisis o interpretación de los datos.

b).- Participar en el diseño del trabajo de investigación o en la revisión crítica de su contenido intelectual.

c).- Aprobar la versión final del artículo que s epublica

d). Estar en capacidad de responder acerca de la exactitud, integridad y validez de cualquier parte del trabajo".

\section{Referencias}

1. Castaño-Vergara DM, Cardona-Arango D. Percepción del estado de salud y factores asociados en adultos mayores. Rev Salud Pública. 2015;17(2):171-83. DoI: http://dx.doi.org/10.15446/rsap. v17n2.30730

2. Torres Palma W, Flores Galaz M. Factores predictores del bienestar subjetivo en adultos mayores. Rev Psico. 2018;36(1):9-48. DoI: https://doi.org/10.18800/psico.201801.001

3. Teixeira JS, Corrêa JC, Rafael CBS, et al. Envelhecimento e percepção corporal de idosos institucionalizados. Rev Bras Geriatr Gerontol. 2012;15(1):63-8. DoI: https://doi.org/10.1590/S180998232012000100007

4. Confortin-Cararo S, Giehl-Corseuil MW, Antes-Ledur D, et al. Autopercepção positiva de saúde em idosos: estudo populacional no Sul do Brasil. Cad. Saúde Pública. 2015;31(5):1049-60. DoI: https://doi.org/10.1590/0102-311X00132014

5. Peláez E, Acosta LD, Carrizo ED. Factores asociados a la autopercepción de salud en adultos mayores. Rev Cubana Salud Pública. [internet]. 2015 [citado 2020 oct. 4]; 41(4):638-48. Disponible en: http://www.revsaludpublica.sld.cu/index.php/spu/article/view/516

6. Vargas-Santillán ML, Arana-Gómez B, García-Hernández ML, et al. Significado de salud: la vivencia del adulto mayor. Aquichan. 2017;17(2):171-82. DoI: http://dx.doi.org/10.5294/ aqui.2017.17.2.6

7. Ebrahimi Z, Wilhelmson K, et al. Frail elders' experiences with and perceptions of health. Qual Health Res. 2012;22(11):1513-23. DOI: https://doi.org/10.1177/1049732312457246
8. Komatsu H, Yagasaki K, et al. Regular group exercise contributes to balanced health in older adults in Japan: A qualitative study. BMC Geriatr. 2017;(17):190. DoI: https://doi.org/10.1186/s12877017-0584-3

9. Troncoso-Pantoja C, Valdebenito-Mardones M. Interpretación de la percepción en la realización de actividad física para un grupo de adultos mayores. Index Enferm. [internet]. 2019 [citado 2020 oct. 5]; 28(1-2):33-36. Disponible en: http:// scielo.isciii.es/scielo.php?script $=$ sci_arttext\&pid $=\mathrm{S} 1132$ $12962019000100007 \& \operatorname{lng}=$ es $12962019000100007 \& \operatorname{lng}=$ es

10. Jancey JM, Clarke A, Howat P, et al. Perceptions of physical activity by older adults: A qualitative study. Health Education Journal. 2009;68(3):196-206. DOI: https://doi. org/10.1177/0017896909339531

11. Lera-López F, Garrues-Irisarri MA, Ollo-López A, et al. Actividad física y salud autopercibida en personas mayores de 50 años. Rev Int Med Cienc Act Fís Deporte. 2017;17(67):559-71. Dor: https:// doi.org/10.15366/rimcafd2017.67.011

12. Cerquera-Córdoba AM, Flórez-Jaimes LO, Linares-Restrepo MM. Autopercepción de la salud en el adulto mayor. Revista Virtual Universidad Católica del Norte [internet]. 2010 [citado 2020 oct. 2]; (31):407-28. Disponible en: https://www.redalyc.org/articulo.oa?id=194214587018

13. Beyer AK. Wolff JK, Warner LM, et al. The role of physical activity in the relationship between self-perceptions of ageing and self-rate health in older adults. Psychol Health. 2015;30(6):67185. DoI: https://doi.org/10.1080/08870446.2015.1014370

14. Dantas GD, Moreira V. El método fenomenológico crítico de investigación con Base en el pensamiento de Merleau Ponty. Ter Psicol. 2009;27(2):247-57.

15. Perruchoud González S. La fenomenología según Merleau-Ponty: un camino de descenso hacia las cosas. Revista de Filosofía, 2017;42(1):59-76. DoI: http://dx.doi.org/10.5209/RESF.55447

16. Colombia, Ministerio de Salud y Protección Social. Política colombiana de envejecimiento humano y vejez 2015-2024. Bogotá; 2015.

17. Chetty S. The case study method for research in small and medium sized firms. Int Small Bus J. 1996; 15(1):73-85. Dor: https:// doi.org/10.1177/0266242696151005

18. Ponty M. Fenomenología de la percepción. Barcelona: Planeta de Agostini S. A.; 1993.

19. Cleverbridge AG. Atlas.ti . Qualitative data analysis. Version Windows 8 [software]. 2017. [citado 2020 oct. 1]. Disponible en: https://atlasti.cleverbridge.com/74/pl/1120380213w7vTYhSpCVFhnnNSG2L-1-2-1

20. Taylor SJ, Bogdan R. Introducción a los métodos cualitativos de investigación. Barcelona: Paidós; 1990, vol. 1.

21. Asociación Médica Mundial (AMM). Declaración de Helsinki de la AMM. Principios éticos para las investigaciones éticas en los seres humanos. 64 Asamblea General, Fortaleza, Brasil, octubre de 2013. Disponible en: https://www.wma.net/es/policies-post/ declaracion-de-helsinki-de-la-amm-principios-eticos-para-lasinvestigaciones-medicas-en-seres-humanos/

22. Colombia, Ministerio de Salud. Resolución 8430, por la cual se establecen las normas científicas, técnicasy administrativas para la investigación en salud (1993, oct. 4).

23. Song M, Kong E. Older adults' definitions of health: A metasynthesis. Inter J Nurs Stud. 2015;52(6):1097-1106. DoI: http:// dx.doi.org/10.1016/j.ijnurstu.2015.02.001

24. López-Benavente Y, Arnau-Sánchez J, Ros-Sánchez T, et al. Dificultades y motivaciones para el ejercicio físico en mujeres mayores de 65 años. Un estudio cualitativo. Rev Latino-Am. 
Enfermagem. 2018;26e2989. DoI: https://doi.org/10.1590/15188345.2392.2989

25. Tuohy D, Cooney A. Older women's experiences of aging and health: An interpretive phenomenological study. Gerontol Geriatr Med. 2019;(5):1-10. DoI: https://doi org $/ 10.1177 / 2333721419834308$

26. Seah B, Espnes GA, Ang ENK, et al. Achieving healthy ageing through the perspective of sense of coherence among senioronly households: A qualitative study. Aging Ment Health 2020;25(5):936-45. DoI: https://doi.org/10.1080/13607863.2020.1 725805
27. Wondolowski C, Davis K. The lived experience of health in the oldes old: A phenomenological study. Nursing Science Quarterly. 1991;4(3):113-18. DoI: https://doi. org $/ 10.1177 / 089431849100400308$

28. López-Oviedo CL. Aproximaciones teóricas para un estudio acerca del sentido del lugar en parques urbanos. Rev. de Invest. Soc. 2013;10(16):31-53. http://ru.iis.sociales.unam.mx/jspui/bitstream/IIS/5365/1/02_lopez.pdf 